\title{
A note on the periodic $L_{2}$-discrepancy of Korobov's $p$-sets
}

\author{
Josef Dick, Aicke Hinrichs, and Friedrich Pillichshammerid
}

\begin{abstract}
We study the periodic $L_{2}$-discrepancy of point sets in the $d$ dimensional torus. This discrepancy is intimately connected with the root-mean-square $L_{2}$-discrepancy of shifted point sets, with the notion of diaphony, and with the worst-case error of cubature formulas for the integration of periodic functions in Sobolev spaces of mixed smoothness. In discrepancy theory, many results are based on averaging arguments. In order to make such results relevant for applications, one requires explicit constructions of point sets with "average" discrepancy. In our main result, we study Korobov's $p$-sets and show that this point sets have periodic $L_{2}$-discrepancy of average order. This result is related to an open question of Novak and Woźniakowski.
\end{abstract}

Mathematics Subject Classification. 11K38.

Keywords. Korobov p-set, Quasi-Monte Carlo, Periodic discrepancy, Tractability.

1. Introduction. In this note, we study the periodic $L_{2}$-discrepancy which is a quantitative measure for the irregularity of distribution of a point set, but which is also closely related to the worst-case integration error of quasi-Monte Carlo integration rules (see, for example, $[3-5,9]$ ). In order to state its definition, we first explain the "test-sets" that are considered in this specific notion of discrepancy.

For $x, y \in[0,1)$, we define the periodic "interval" $I(x, y)$ as

$$
I(x, y)= \begin{cases}{[x, y)} & \text { if } x \leq y \\ {[0, y) \cup[x, 1)} & \text { if } x>y\end{cases}
$$

For dimension $d>1$ and $\boldsymbol{x}=\left(x_{1}, \ldots, x_{d}\right)$ and $\boldsymbol{y}=\left(y_{1}, \ldots, y_{d}\right)$ in $[0,1)^{d}$, the periodic "boxes" $B(\boldsymbol{x}, \boldsymbol{y})$ are given by

$$
B(\boldsymbol{x}, \boldsymbol{y})=I\left(x_{1}, y_{1}\right) \times \cdots \times I\left(x_{d}, y_{d}\right) .
$$


The local discrepancy of a point set $\mathcal{P}=\left\{\boldsymbol{x}_{1}, \boldsymbol{x}_{2}, \ldots, \boldsymbol{x}_{N}\right\}$ consisting of $N$ elements in the $d$-dimensional unit cube with respect to a periodic box $B=B(\boldsymbol{x}, \boldsymbol{y})$ is given by

$$
\Delta_{\mathcal{P}}(B)=\frac{\#\left\{j \in\{1, \ldots, N\}: \boldsymbol{x}_{j} \in B\right\}}{N}-\operatorname{volume}(B) .
$$

Then the periodic $L_{2}$-discrepancy of $\mathcal{P}$ is the $L_{2}$-norm of the local discrepancy taken over all periodic boxes $B=B(\boldsymbol{x}, \boldsymbol{y})$, i.e.,

$$
L_{2, N}^{\mathrm{per}}(\mathcal{P})=\left(\int_{[0,1]^{d}} \int_{[0,1]^{d}} \Delta_{\mathcal{P}}(B(\boldsymbol{x}, \boldsymbol{y}))^{2} \mathrm{~d} \boldsymbol{x} \mathrm{d} \boldsymbol{y}\right)^{1 / 2}
$$

The usual $L_{2}$-discrepancy of a point set $\mathcal{P}$ is defined as

$$
L_{2, N}(\mathcal{P})=\left(\int_{[0,1]^{d}} \Delta_{\mathcal{P}}(B(\mathbf{0}, \boldsymbol{y}))^{2} \mathrm{~d} \boldsymbol{y}\right)^{1 / 2} .
$$

In the closely connected context of worst-case errors for cubature formulas, it is natural to extend these notions to weighted point sets. If, additionally to the point set $\mathcal{P}=\left\{\boldsymbol{x}_{1}, \boldsymbol{x}_{2}, \ldots, \boldsymbol{x}_{N}\right\}$, we also have a set of associated real weights $\boldsymbol{w}=\left\{w_{1}, w_{2}, \ldots, w_{N}\right\}$, the local discrepancy of the weighted point set $\mathcal{P}$ is given as

$$
\Delta_{\mathcal{P}}(B, \boldsymbol{w})=\left(\sum_{j: x_{j} \in B} w_{j}\right)-\operatorname{volume}(B) .
$$

Then $\Delta_{\mathcal{P}}(B)=\Delta_{\mathcal{P}}(B, \boldsymbol{w})$ is obtained for equal weights $w_{j}=1 / N$. The periodic $L_{2}$-discrepancy of the weighted point set $\mathcal{P}$ is

$$
L_{2, N}^{\mathrm{per}}(\mathcal{P}, \boldsymbol{w})=\left(\int_{[0,1]^{d}} \int_{[0,1]^{d}} \Delta_{\mathcal{P}}(B(\boldsymbol{x}, \boldsymbol{y}), \boldsymbol{w})^{2} \mathrm{~d} \boldsymbol{x} \mathrm{d} \boldsymbol{y}\right)^{1 / 2} .
$$

The usual $L_{2}$-discrepancy of the weighted point set $\mathcal{P}$ is

$$
L_{2, N}(\mathcal{P}, \boldsymbol{w})=\left(\int_{[0,1]^{d}} \Delta_{\mathcal{P}}(B(\mathbf{0}, \boldsymbol{y}), \boldsymbol{w})^{2} \mathrm{~d} \boldsymbol{y}\right)^{1 / 2}
$$

For a (weighted) point set $\mathcal{P}=\left\{\boldsymbol{x}_{1}, \boldsymbol{x}_{2}, \ldots, \boldsymbol{x}_{N}\right\}$ and a real vector $\boldsymbol{\delta} \in$ $[0,1)^{d}$, the shifted point set $\mathcal{P}+\boldsymbol{\delta}$ is defined as

$$
\mathcal{P}+\boldsymbol{\delta}=\left\{\left\{\boldsymbol{x}_{1}+\boldsymbol{\delta}\right\},\left\{\boldsymbol{x}_{2}+\boldsymbol{\delta}\right\}, \ldots,\left\{\boldsymbol{x}_{N}+\boldsymbol{\delta}\right\}\right\},
$$

where $\left\{\boldsymbol{x}_{j}+\boldsymbol{\delta}\right\}$ means that the fractional-part-function, $\{x\}=x-\lfloor x\rfloor$ for non-negative real numbers $x$, is applied component-wise to the vector $\boldsymbol{x}_{j}+\boldsymbol{\delta}$. 
The root-mean-square $L_{2}$-discrepancy of a shifted (and weighted) point set $\mathcal{P}$ with respect to all uniformly distributed shift vectors $\delta \in[0,1)^{d}$ is

$$
\sqrt{\mathbb{E}_{\delta}\left[\left(L_{2, N}(\mathcal{P}+\boldsymbol{\delta}, \boldsymbol{w})\right)^{2}\right]}=\left(\int_{[0,1]^{d}}\left(L_{2, N}(\mathcal{P}+\boldsymbol{\delta}, \boldsymbol{w})\right)^{2} \mathrm{~d} \boldsymbol{\delta}\right)^{1 / 2} .
$$

Note that

$$
\mathbb{E}_{\boldsymbol{\delta}}\left[L_{2, N}(\mathcal{P}+\boldsymbol{\delta}, \boldsymbol{w})\right] \leq \sqrt{\mathbb{E}_{\boldsymbol{\delta}}\left[\left(L_{2, N}(\mathcal{P}+\boldsymbol{\delta}, \boldsymbol{w})\right)^{2}\right]}
$$

If the weights are the standard equal weights $w_{j}=1 / N$, we drop $\boldsymbol{w}$ from the notation.

The following relation between periodic $L_{2}$-discrepancy and root-meansquare $L_{2}$-discrepancy of a shifted point set $\mathcal{P}$ holds (see also [9]):

Proposition 1. For $\mathcal{P}=\left\{\boldsymbol{x}_{1}, \boldsymbol{x}_{2}, \ldots, \boldsymbol{x}_{N}\right\}$ in $[0,1)^{d}$ and weights $\boldsymbol{w}=\left\{w_{1}, w_{2}\right.$, $\left.\ldots, w_{N}\right\}$, we have

$$
L_{2, N}^{\mathrm{per}}(\mathcal{P}, \boldsymbol{w})=\sqrt{\mathbb{E}_{\boldsymbol{\delta}}\left[\left(L_{2, N}(\mathcal{P}+\boldsymbol{\delta}, \boldsymbol{w})\right)^{2}\right]} .
$$

In particular, we have

$$
L_{2, N}^{\text {per }}(\mathcal{P})=\sqrt{\mathbb{E}_{\delta}\left[\left(L_{2, N}(\mathcal{P}+\boldsymbol{\delta})\right)^{2}\right]} .
$$

Proof. We write down the proof for dimension $d=1$ and the equal weighted case only. The general case follows by similar arguments. We have

$$
\begin{aligned}
\mathbb{E}_{\delta}\left[\left(L_{2, N}(\mathcal{P}+\delta)\right)^{2}\right] & =\int_{0}^{1} \int_{0}^{1} \Delta_{\mathcal{P}+\delta}([0, y))^{2} \mathrm{~d} y \mathrm{~d} \delta \\
& =\int_{0}^{1}\left(\int_{0}^{y} \Delta_{\mathcal{P}+\delta}([0, y))^{2} \mathrm{~d} \delta+\int_{y}^{1} \Delta_{\mathcal{P}+\delta}([0, y))^{2} \mathrm{~d} \delta\right) \mathrm{d} y .
\end{aligned}
$$

We consider two cases:

- If $\delta<y$, then $\left\{x_{j}+\delta\right\} \in[0, y)$ iff $x_{j}+\delta \in[0, y)$ or $x_{j}+\delta-1 \in[0, y)$ and this holds iff $x_{j} \in[0, y-\delta) \cup[1-\delta, 1)$. Hence

$$
\left\{x_{j}+\delta\right\} \in[0, y) \Leftrightarrow x_{j} \in I(1-\delta, y-\delta) .
$$

Note that volume $(I(1-\delta, y-\delta))=y$.

- If $\delta \geq y$, then $\left\{x_{j}+\delta\right\} \in[0, y)$ iff $x_{j}+\delta \in[0, y)$ or $x_{j}+\delta-1 \in[0, y)$ and this holds iff $x_{j} \in[1-\delta, 1+y-\delta)$. Hence

$$
\left\{x_{j}+\delta\right\} \in[0, y) \Leftrightarrow x_{j} \in I(1-\delta, 1+y-\delta) .
$$

Note that volume $(I(1-\delta, 1+y-\delta))=y$. 
Hence

$$
\begin{aligned}
\mathbb{E}_{\delta} & {\left[\left(L_{2, N}(\mathcal{P}+\delta)\right)^{2}\right] } \\
& =\int_{0}^{1}\left(\int_{0}^{y} \Delta_{\mathcal{P}}(I(1-\delta, y-\delta))^{2} \mathrm{~d} \delta+\int_{y}^{1} \Delta_{\mathcal{P}}(I(1-\delta, 1+y-\delta))^{2} \mathrm{~d} \delta\right) \mathrm{d} y \\
& =\int_{0}^{1}\left(\int_{1-y}^{1} \Delta_{\mathcal{P}}(I(t, y+t-1))^{2} \mathrm{~d} t+\int_{0}^{1-y} \Delta_{\mathcal{P}}(I(t, y+t))^{2} \mathrm{~d} t\right) \mathrm{d} y \\
& =\int_{0}^{1} \int_{1-t}^{1} \Delta_{\mathcal{P}}(I(t, y+t-1))^{2} \mathrm{~d} y \mathrm{~d} t+\int_{0}^{1} \int_{0}^{1-t} \Delta_{\mathcal{P}}(I(t, y+t))^{2} \mathrm{~d} y \mathrm{~d} t \\
& =\int_{0}^{1} \int_{0}^{t} \Delta_{\mathcal{P}}(I(t, z))^{2} \mathrm{~d} z \mathrm{~d} t+\int_{0}^{1} \int_{t}^{1}(I(t, z))^{2} \mathrm{~d} z \mathrm{~d} t \\
& =\int_{0}^{1} \int_{0}^{1} \Delta_{\mathcal{P}}(I(t, z))^{2} \mathrm{~d} z \mathrm{~d} t \\
& =\left(L_{2, N}^{\text {per }}(\mathcal{P})\right)^{2},
\end{aligned}
$$

where we just applied several elementary substitutions.

Another important fact is that the periodic $L_{2}$-discrepancy can be expressed in terms of exponential sums.

Proposition 2. We have

$$
\left(L_{2, N}^{\text {per }}(\mathcal{P})\right)^{2}=\frac{1}{3^{d}} \sum_{\boldsymbol{k} \in \mathbb{Z}^{d} \backslash\{\mathbf{0}\}} \frac{1}{r(\boldsymbol{k})^{2}}\left|\frac{1}{N} \sum_{h=1}^{N} \exp \left(2 \pi i \boldsymbol{k} \cdot \boldsymbol{x}_{h}\right)\right|^{2},
$$

where $i=\sqrt{-1}$ and where for $\boldsymbol{k}=\left(k_{1}, \ldots, k_{d}\right) \in \mathbb{Z}^{d}$, we set

$$
r(\boldsymbol{k})=\prod_{j=1}^{d} r\left(k_{j}\right) \quad \text { and } \quad r\left(k_{j}\right)= \begin{cases}1 & \text { if } k_{j}=0, \\ \frac{2 \pi\left|k_{j}\right|}{\sqrt{6}} & \text { if } k_{j} \neq 0 .\end{cases}
$$

Proof. See [4, p. 390].

The above formula shows that the periodic $L_{2}$-discrepancy is - up to a multiplicative factor - exactly the diaphony which is a well-known measure for the irregularity of the distribution of point sets and which was introduced by Zinterhof [15] in the year 1976.

In Section 2, we will estimate the periodic $L_{2}$-discrepancy of certain types of point sets that recently gained some attention in the context of error bounds with favourable weak dependence on the dimension $d$ (see $[1,2]$ ). On the other hand, we also show in Section 3 that even the weighted version of the periodic $L_{2}$-discrepancy, when considered in the normalized setting, suffers from the curse of dimensionality. 
2. The periodic $\boldsymbol{L}_{\mathbf{2}}$-discrepancy of Korobov's $\boldsymbol{p}$-sets. Let $p$ be a prime number. We consider the following point sets in $[0,1)^{d}$ :

- Let $\mathcal{P}_{p}^{\text {Kor }}=\left\{\boldsymbol{x}_{0}, \ldots, \boldsymbol{x}_{p-1}\right\}$ with

$$
\boldsymbol{x}_{n}=\left(\left\{\frac{n}{p}\right\},\left\{\frac{n^{2}}{p}\right\}, \ldots,\left\{\frac{n^{d}}{p}\right\}\right) \text { for } n=0,1, \ldots, p-1 .
$$

The point set $\mathcal{P}_{p}^{\text {Kor }}$ was introduced by Korobov [8] (see also [6, Section 4.3]).

- Let $\mathcal{Q}_{p^{2}}^{\text {Kor }}=\left\{\boldsymbol{x}_{0}, \ldots, \boldsymbol{x}_{p^{2}-1}\right\}$ with

$$
\boldsymbol{x}_{n}=\left(\left\{\frac{n}{p^{2}}\right\},\left\{\frac{n^{2}}{p^{2}}\right\}, \ldots,\left\{\frac{n^{d}}{p^{2}}\right\}\right) \quad \text { for } n=0,1, \ldots, p^{2}-1 .
$$

The point set $\mathcal{Q}_{p^{2}}^{\text {Kor }}$ was introduced by Korobov [7] (see also [6, Section 4.3]).

- Let $\mathcal{R}_{p^{2}}^{\mathrm{Kor}}=\left\{\boldsymbol{x}_{a, k}: a, k \in\{0, \ldots, p-1\}\right\}$ with

$$
\boldsymbol{x}_{a, k}=\left(\left\{\frac{k}{p}\right\},\left\{\frac{a k}{p}\right\}, \ldots,\left\{\frac{a^{d-1} k}{p}\right\}\right) \quad \text { for } a, k=0,1, \ldots, p-1 \text {. }
$$

Note that $\mathcal{R}_{p^{2}}^{\text {Kor }}$ is the multi-set union of all Korobov lattice point sets with modulus $p$. The point set $\mathcal{R}_{p^{2}}^{\text {Kor }}$ was introduced by Hua and Wang (see [6, Section 4.3]).

Hua and Wang [6] called the point sets $\mathcal{P}_{p}^{\text {Kor }}, \mathcal{Q}_{p^{2}}^{\text {Kor }}$, and $\mathcal{R}_{p^{2}}^{\text {Kor }}$ the $p$-sets. We have

$$
\left|\mathcal{P}_{p}^{\mathrm{Kor}}\right|=p \quad \text { and } \quad\left|\mathcal{Q}_{p^{2}}^{\mathrm{Kor}}\right|=\left|\mathcal{R}_{p^{2}}^{\mathrm{Kor}}\right|=p^{2} .
$$

Theorem 1. For Korobov's p-sets $\mathcal{P} \in\left\{\mathcal{P}_{p}^{\text {Kor }}, \mathcal{Q}_{p^{2}}^{\text {Kor }}, \mathcal{R}_{p^{2}}^{\text {Kor }}\right\}$, we have

$$
L_{2, N}^{\mathrm{per}}(\mathcal{P}) \leq \frac{d}{2^{d / 2}} \frac{1}{\sqrt{N}}
$$

For the proof of Theorem 1, we need the following lemma.

Lemma 1. Let $p$ be a prime number and let $d \in \mathbb{N}$. Then for all $h_{1}, \ldots, h_{d} \in \mathbb{Z}$ such that $p \nmid h_{j}$ for at least one $j \in\{1,2, \ldots, d\}$, we have

$$
\begin{aligned}
& \left|\sum_{n=0}^{p-1} \exp \left(2 \pi i\left(h_{1} n+h_{2} n^{2}+\cdots+h_{d} n^{d}\right) / p\right)\right| \leq(d-1) \sqrt{p}, \\
& \left|\sum_{n=0}^{p^{2}-1} \exp \left(2 \pi i\left(h_{1} n+h_{2} n^{2}+\cdots+h_{d} n^{d}\right) / p^{2}\right)\right| \leq(d-1) p . \\
& \left|\sum_{a=0}^{p-1} \sum_{k=0}^{p-1} \exp \left(2 \pi i k\left(h_{1}+h_{2} a+\cdots+h_{d} a^{d-1}\right) / p\right)\right| \leq(d-1) p .
\end{aligned}
$$

Proof. Eq. (1) follows from a bound from A. Weil [14] on exponential sums which is widely known as Weil bound (see also [10, Theorem 5.38]). For details, we refer to [1]. For a proof of Eq. (2), we refer to [6, Lemma 4.6]. A proof of Eq. (3) can be found in [2]. 
Proof of Theorem 1. We use the formula from Proposition 2 for the periodic $L_{2}$-discrepancy and estimate the exponential sum with the help of Lemma 1. We provide the details only for $\mathcal{P}_{p}^{\text {Kor }}$ and $\mathcal{Q}_{p^{2}}^{\text {Kor }}$. The proof for $\mathcal{R}_{p^{2}}^{\text {Kor }}$ is analogous.

Before we start, we mention the following easy results that will be used later in the proof. For $\boldsymbol{k}=\left(k_{1}, \ldots, k_{d}\right) \in \mathbb{Z}^{d}$, we write $N \mid \boldsymbol{k}$ to indicate that $N \mid k_{j}$ for all $j \in\{1,2, \ldots, d\}$. Likewise, $N \nmid \boldsymbol{k}$ indicates that there is at least one index $j \in\{1,2, \ldots, d\}$ with $N \nmid k_{j}$. We have

$$
\sum_{k \in \mathbb{Z}^{d}} \frac{1}{r(\boldsymbol{k})^{2}}=\left(1+2 \sum_{k=1}^{\infty} \frac{6}{4 \pi^{2} k^{2}}\right)^{d}=\left(1+2 \frac{6}{4 \pi^{2}} \frac{\pi^{2}}{6}\right)^{d}=\left(\frac{3}{2}\right)^{d}
$$

and

$$
\begin{aligned}
\sum_{\substack{k \in \mathbb{Z} \\
N \nmid k}} \frac{1}{r(\boldsymbol{k})^{2}} & =\sum_{\boldsymbol{k} \in \mathbb{Z}^{d}} \frac{1}{r(\boldsymbol{k})^{2}}-\sum_{\substack{k \in \mathbb{Z} \\
N \mid \boldsymbol{k}}} \frac{1}{r(\boldsymbol{k})^{2}} \\
& =\left(\frac{3}{2}\right)^{d}-\left(1+2 \sum_{k=1}^{\infty} \frac{6}{4 \pi^{2}(N k)^{2}}\right)^{d} \\
& =\left(\frac{3}{2}\right)^{d}-\left(1+\frac{1}{2 N^{2}}\right)^{d} .
\end{aligned}
$$

First we study $\mathcal{P}_{p}^{\text {Kor }}$. Then $N=\left|\mathcal{P}_{p}^{\text {Kor }}\right|=p$. Using Eq. (1) from Lemma 1, we have

$$
\begin{aligned}
& \sum_{\boldsymbol{k} \in \mathbb{Z}^{d} \backslash\{\mathbf{0}\}} \frac{1}{r(\boldsymbol{k})^{2}}\left|\frac{1}{N} \sum_{h=1}^{N} \exp \left(2 \pi \mathrm{i} \boldsymbol{k} \cdot \boldsymbol{x}_{h}\right)\right|^{2} \\
& \leq \sum_{\substack{k \in \mathbb{Z}^{d} \\
N \nmid k}} \frac{1}{r(\boldsymbol{k})^{2}} \frac{(d-1)^{2}}{N}+\sum_{\substack{k \in \mathbb{Z}^{d} \backslash\{\mathbf{0}\} \\
N \backslash k}} \frac{1}{r(\boldsymbol{k})^{2}}\left|\frac{1}{N} \sum_{h=0}^{N-1} \exp \left(\frac{2 \pi \mathrm{i} \boldsymbol{k} \cdot\left(h, h^{2}, \ldots, h^{d}\right)}{N}\right)\right|^{2} \\
& =\frac{(d-1)^{2}}{N} \sum_{\substack{k \in \mathbb{Z}^{d} \\
N \nmid k}} \frac{1}{r(\boldsymbol{k})^{2}}+\sum_{\boldsymbol{k} \in \mathbb{Z}^{d} \backslash\{\mathbf{0}\}} \frac{1}{r(N \boldsymbol{k})^{2}} \\
& =\frac{(d-1)^{2}}{N}\left(\left(\frac{3}{2}\right)^{d}-\left(1+\frac{1}{2 N^{2}}\right)^{d}\right)+\left(1+\frac{1}{2 N^{2}}\right)^{d}-1 \\
& \leq \frac{(d-1)^{2}}{N}\left(\frac{3}{2}\right)^{d}+\frac{1}{N^{2}}\left(\frac{3}{2}\right)^{d} \\
& \leq \frac{d^{2}}{N}\left(\frac{3}{2}\right)^{d} \cdot
\end{aligned}
$$

Inserting this bound into the formula given in Proposition 2 gives

$$
\left(L_{2, N}^{\text {per }}\left(\mathcal{P}_{p}^{\text {Kor }}\right)\right)^{2} \leq \frac{1}{3^{d}} \frac{d^{2}}{N}\left(\frac{3}{2}\right)^{d}=\frac{d^{2}}{2^{d}} \frac{1}{N} .
$$

This yields the desired result for $\mathcal{P}_{p}^{\text {Kor }}$. 
Now we turn to $\mathcal{Q}_{p^{2}}^{\text {Kor }}$. Here $N=\left|\mathcal{Q}_{p^{2}}^{\text {Kor }}\right|=p^{2}$. Using Eq. (2) from Lemma 1, we have

$$
\begin{aligned}
& \sum_{\substack{\boldsymbol{k} \in \mathbb{Z}^{d} \backslash\{\mathbf{0}\}\\
}} \frac{1}{r(\boldsymbol{k})^{2}}\left|\frac{1}{N} \sum_{h=1}^{N} \exp \left(2 \pi \mathrm{i} \boldsymbol{k} \cdot \boldsymbol{x}_{h}\right)\right|^{2} \\
& \leq \sum_{\substack{\boldsymbol{k} \in \mathbb{Z}^{d} \\
p \nmid k}} \frac{1}{r(\boldsymbol{k})^{2}} \frac{(d-1)^{2}}{N}+\sum_{\substack{k \in \mathbb{Z}^{d} \backslash\{\mathbf{0}\} \\
p \mid k}} \frac{1}{r(\boldsymbol{k})^{2}}\left|\frac{1}{N} \sum_{h=0}^{N-1} \exp \left(\frac{2 \pi \mathrm{i} \boldsymbol{k} \cdot\left(h, h^{2}, \ldots, h^{d}\right)}{N}\right)\right|^{2} \\
& \leq \sum_{\substack{k \in \mathbb{Z}^{d} \\
p \nmid k}} \frac{1}{r(\boldsymbol{k})^{2}} \frac{(d-1)^{2}}{N}+\sum_{\substack{\boldsymbol{k} \in \mathbb{Z}^{d} \backslash\{\mathbf{0}\}\\
}} \frac{1}{r(p \boldsymbol{k})^{2}} \\
& \leq \frac{(d-1)^{2}}{N}\left(\left(\frac{3}{2}\right)^{d}-\left(1+\frac{1}{2 p^{2}}\right)^{d}\right)+\frac{1}{p^{2}}\left(\left(\frac{3}{2}\right)^{d}-1\right) \\
& \leq \frac{d^{2}}{N}\left(\frac{3}{2}\right)^{d} .
\end{aligned}
$$

Hence

$$
\left(L_{2, N}^{\text {per }}\left(\mathcal{Q}_{p^{2}}^{\text {Kor }}\right)\right)^{2} \leq \frac{d^{2}}{2^{d}} \frac{1}{N} .
$$

This yields the desired result for $\mathcal{Q}_{p^{2}}^{\text {Kor }}$.

In order to prove the bound on $L_{2, N}^{\text {per }}\left(\mathcal{R}_{p^{2}}^{\text {Kor }}\right)$, use Eq. (3) from Lemma 1.

Remark 1. Note that the dependence on the dimension of our bound on the periodic $L_{2}$-discrepancy is only $d / 2^{d / 2}$, which looks very promising regarding tractability properties at first sight. However, it can be easily checked that already the periodic $L_{2}$-discrepancy of the empty set is only $3^{-d / 2}$ (see the forthcoming Lemma 2) which is much smaller than $d / 2^{d / 2}$. We will see in the next section that the periodic $L_{2}$-discrepancy considered in the normalized setting suffers from the curse of dimensionality.

In discrepancy theory, many results are based on averaging arguments. In order to make such results relevant for applications, one requires explicit constructions of point sets with "average" discrepancy. It is easily checked that the average squared periodic $L_{2}$-discrepancy

$$
\operatorname{avg}_{2}^{\text {per }}(N, d):=\left(\int_{[0,1]^{d}} \ldots \int_{[0,1]^{d}}\left(L_{2, N}^{\text {per }}\left(\left\{\boldsymbol{x}_{1}, \ldots, \boldsymbol{x}_{N}\right\}\right)\right)^{2} \mathrm{~d} \boldsymbol{x}_{1} \ldots \mathrm{d} \boldsymbol{x}_{N}\right)^{1 / 2}
$$

equals

$$
\frac{1}{\sqrt{N}}\left(\frac{1}{2^{d}}-\frac{1}{3^{d}}\right)^{1 / 2} .
$$

Hence Theorem 1 shows that the periodic $L_{2}$-discrepancy of Korobov's $p$-set is almost of average order. 
Remark 2. There is some relation to [12, Open Problem 40, p. 57]. There the authors ask for a construction of an $N$-element point set in dimension $d$ in time polynomial in $N$ and $d$ for which the $L_{p}$-discrepancy is less than the average $L_{p}$-discrepancy taken over all $N$-element point sets in dimension $d$. Here we have - up to a linear factor $d$ - the answer to this question for the periodic $L_{2}$-discrepancy.

Remark 3. Our result also gives some information about the usual $L_{2^{-}}$ discrepancy: The average $L_{2}$-discrepancy is

$$
\operatorname{avg}_{2}(N, d):=\left(\int_{[0,1]^{d}} \ldots \int_{[0,1]^{d}}\left(L_{2, N}\left(\left\{\boldsymbol{x}_{1}, \ldots, \boldsymbol{x}_{N}\right\}\right)\right)^{2} \mathrm{~d} \boldsymbol{x}_{1} \ldots \mathrm{d} \boldsymbol{x}_{N}\right)^{1 / 2}
$$

and this is well-known to also be

$$
\frac{1}{\sqrt{N}}\left(\frac{1}{2^{d}}-\frac{1}{3^{d}}\right)^{1 / 2} .
$$

Using Proposition 1, we obtain that the root-mean-square $L_{2}$-discrepancy of the shifted $p$-sets is at most $\frac{d}{2^{d / 2}} \frac{1}{\sqrt{N}}$ and hence almost of average order. This also implies that there must exist a shift $\boldsymbol{\delta}^{*} \in[0,1)$ for the $p$-sets $\mathcal{P} \in\left\{\mathcal{P}_{p}^{\text {Kor }}, \mathcal{Q}_{p^{2}}^{\text {Kor }}, \mathcal{R}_{p^{2}}^{\text {Kor }}\right\}$ such that

$$
L_{2, N}\left(\mathcal{P}+\boldsymbol{\delta}^{*}\right) \leq \frac{d}{2^{d / 2}} \frac{1}{\sqrt{N}} .
$$

Whether the $L_{2}$-discrepancy of the $p$-sets itself satisfies the bound $\frac{d}{2^{d / 2}} \frac{1}{\sqrt{N}}$ is an interesting open problem.

3. The curse of dimensionality for the periodic $L_{2}$-discrepancy in the normalized setting. In this section, we are interested in the inverse $N_{2}^{\text {per }}(\varepsilon, d)$ of the periodic $L_{2}$-discrepancy which is the minimal number $N \in \mathbb{N}$ for which there exists an $N$-element point set $\mathcal{P}$ in $[0,1)^{d}$ whose periodic $L_{2}$-discrepancy is less then $\varepsilon$ times the initial periodic $L_{2}$-discrepancy. Since lower bounds become stronger if proved also for the weighted version, we consider here also the inverse of the weighted periodic $L_{2}$-discrepancy $N_{2}^{\text {per,w }}(\varepsilon, d)$ and compare the results to the results for the unweighted case and to the case where only positive weights are allowed.

Lemma 2. The initial periodic $L_{2}$-discrepancy is

$$
L_{2,0}^{\text {per }}=\left(\int_{[0,1]^{d}} \int_{[0,1]^{d}} \operatorname{volume}(B(\boldsymbol{x}, \boldsymbol{y}))^{2} \mathrm{~d} \boldsymbol{x} \mathrm{d} \boldsymbol{y}\right)^{1 / 2}=\frac{1}{3^{d / 2}} .
$$

We omit the easy proof of this lemma.

Hence, for $\varepsilon \in(0,1)$ and $d \in \mathbb{N}$,

$N_{2}^{\text {per }}(\varepsilon, d)=\min \left\{N \in \mathbb{N}: \exists \mathcal{P} \subseteq[0,1)^{d},|\mathcal{P}|=N\right.$, and $\left.L_{2, N}^{\text {per }}(\mathcal{P}) \leq \frac{\varepsilon}{3^{d / 2}}\right\}$. 
Analogously, we define $N_{2}^{\text {per,w }}(\varepsilon, d)$ and $N_{2}^{\text {per,w+ }}(\varepsilon, d)$ if arbitrary or only positive weights are allowed, respectively.

In information based complexity, one is particularly interested in the dependence of the inverse discrepancy on $\varepsilon^{-1}$ and $d$. In particular, a polynomial dependence on $d$ would be favourable (see, e.g., [12]). This however is not achievable in the case of the periodic $L_{2}$-discrepancy. Indeed, the following theorem shows that the periodic $L_{2}$-discrepancy suffers from the curse of dimensionality.

Theorem 2. For $\varepsilon \in(0,1)$ and $d \in \mathbb{N}$, we have

$$
N_{2}^{\text {per }}(\varepsilon, d) \geq \frac{1}{1+\varepsilon^{2}}\left(\frac{3}{2}\right)^{d} \quad \text { and } \quad N_{2}^{\text {per,w+ }}(\varepsilon, d) \geq\left(1-\varepsilon^{2}\right)\left(\frac{3}{2}\right)^{d} .
$$

Moreover, for any $\varepsilon_{0} \in(0,1)$, there exists $c>0$ such that

$$
N_{2}^{\mathrm{per}, \mathrm{w}}(\varepsilon, d) \geq c 1.0628^{d}
$$

for all $d \in \mathbb{N}$ and $\varepsilon \in\left(0, \varepsilon_{0}\right)$.

Proof. The lower bound for $N_{2}^{\text {per,w }}(\varepsilon, d)$ follows directly from the corresponding lower bound for the inverse of the non-periodic $L_{2}$-discrepancy, see [11] or $[12,(9.17)]$, together with Proposition 1. Similarly, a lower bound

$$
N_{2}^{\text {per }}(\varepsilon, d) \geq N_{2}^{\text {per,w+ }}(\varepsilon, d) \geq\left(1-\varepsilon^{2}\right) 1.125^{d}
$$

follows from the corresponding lower bound for the inverse of the non-periodic $L_{2}$-discrepancy, see [13] or [12, (9.16)], together with Proposition 1.

To prove the better lower bound for $N_{2}^{\text {per }}(\varepsilon, d)$, we observe that according to $[4$, p. 389-390], we have

$$
L_{2, N}^{\text {per }}(\mathcal{P})^{2}=-\frac{1}{3^{d}}+\frac{1}{N^{2}} \sum_{n, m=1}^{N} \prod_{j=1}^{d}\left(\frac{1}{3}+B_{2}\left(\left|x_{n, j}-x_{m, j}\right|\right)\right),
$$

where $B_{2}(x)$ is the second Bernoulli polynomial, $B_{2}(x)=x^{2}-x+\frac{1}{6}$, and $x_{n, j}$ is the $j^{\text {th }}$ coordinate of the point $\boldsymbol{x}_{n}$. Note that $B_{2}(0)=1 / 6$ and for $x \in[0,1]$, we have $B_{2}(x) \geq B_{2}(1 / 2)=-1 / 12$. Now we have

$$
L_{2, N}^{\text {per }}(\mathcal{P})^{2} \geq-\frac{1}{3^{d}}+\frac{1}{N^{2}} \sum_{\substack{n, m=1 \\ n=m}}^{N} \frac{1}{2^{d}}+\frac{1}{N^{2}} \sum_{\substack{n, m=1 \\ n \neq m}}^{N}\left(\frac{1}{3}-\frac{1}{12}\right)^{d} \geq-\frac{1}{3^{d}}+\frac{1}{N} \frac{1}{2^{d}} \text {. }
$$

Hence

$$
L_{2, N}^{\mathrm{per}}(\mathcal{P}) \leq \frac{\varepsilon}{3^{d / 2}}
$$

implies

$$
-\frac{1}{3^{d}}+\frac{1}{N} \frac{1}{2^{d}} \leq \frac{\varepsilon^{2}}{3^{d}}
$$

and therefore

$$
N \geq \frac{1}{1+\varepsilon^{2}}\left(\frac{3}{2}\right)^{d}
$$


The slightly worse lower bound for $N_{2}^{\text {per,w+ }}(\varepsilon, d)$ follows similarly using

$$
L_{2, N}^{\text {per }}(\mathcal{P}, \boldsymbol{w})^{2}=\frac{1}{3^{d}}-\frac{2}{3^{d}} \sum_{n=1}^{N} w_{n}+\sum_{n, m=1}^{N} w_{n} w_{m} \prod_{j=1}^{d}\left(\frac{1}{3}+B_{2}\left(\left|x_{n, j}-x_{m, j}\right|\right)\right) .
$$

If the weights $w_{n}$ are non-negative, estimating the double sum by the diagonal terms, we have

$$
L_{2, N}^{\text {per }}(\mathcal{P}, \boldsymbol{w})^{2} \geq \frac{1}{3^{d}}-\frac{2}{3^{d}} \sum_{n=1}^{N} w_{n}+\frac{1}{2^{d}} \sum_{n=1}^{N} w_{n}^{2} \geq \frac{1}{3^{d}}-\frac{2^{d} N}{3^{2 d}} .
$$

Now it is easily seen that

$$
L_{2, N}^{\mathrm{per}}(\mathcal{P}, \boldsymbol{w}) \leq \frac{\varepsilon}{3^{d / 2}}
$$

implies

$$
N \geq\left(1-\varepsilon^{2}\right)\left(\frac{3}{2}\right)^{d}
$$

Remark 4. Let $N$ be the smallest prime number larger or equal to

$$
\left\lceil\left(\frac{3}{2}\right)^{d} \frac{d^{2}}{\varepsilon^{2}}\right\rceil=: M
$$

Then it follows from Theorem 1 that for Korobov's $p$-set $\mathcal{P}_{p}^{\text {Kor }}$ with $p=N$, we have

$$
L_{2, N}^{\text {per }}\left(\mathcal{P}_{p}^{\text {Kor }}\right) \leq \frac{\varepsilon}{3^{d / 2}}
$$

and hence

$$
N_{2}^{\text {per,w+ }}(\varepsilon, d) \leq N<2 M=2\left\lceil\left(\frac{3}{2}\right)^{d} \frac{d^{2}}{\varepsilon^{2}}\right\rceil
$$

where we used Bertrand's postulate, which tells us that $M \leq N<2 M$.

This means that the term $3 / 2$ in the lower bounds in Theorem 2 is the exact basis for the exponential dependence of the information complexity in $d$.

Acknowledgements. Open access funding provided by Austrian Science Fund (FWF). This project started while Josef Dick was "Land Oberösterreich Guest Professor" within the Special Research Program "QuasiMonte Carlo Methods: Theory and Applications" which is funded by the Austrian Science Fund (FWF) Project F55-N26, and the government of the Austrian state Upper Austria. It was completed while he visited JKU during a further research stay. A. Hinrichs is supported by the Austrian Science Fund (FWF) Project F5513-N26, which is a part of the Special Research Program "Quasi-Monte Carlo Methods: Theory and Applications".

F. Pillichshammer is supported by the Austrian Science Fund (FWF) Project F5509-N26, which is a part of the Special Research Program "Quasi-Monte Carlo Methods: Theory and Applications". 
Open Access. This article is licensed under a Creative Commons Attribution 4.0 International License, which permits use, sharing, adaptation, distribution and reproduction in any medium or format, as long as you give appropriate credit to the original author(s) and the source, provide a link to the Creative Commons licence, and indicate if changes were made. The images or other third party material in this article are included in the article's Creative Commons licence, unless indicated otherwise in a credit line to the material. If material is not included in the article's Creative Commons licence and your intended use is not permitted by statutory regulation or exceeds the permitted use, you will need to obtain permission directly from the copyright holder. To view a copy of this licence, visit http://creativecommons. org/licenses/by/4.0/.

Publisher's Note Springer Nature remains neutral with regard to jurisdictional claims in published maps and institutional affiliations.

\section{References}

[1] Dick, J.: Numerical integration of Hölder continuous absolutely convergent Fourier-, Fourier cosine-, and Walsh series. J. Approx. Theory 184, 111-145 (2014)

[2] Dick, J., Pillichshammer, F.: The weighted star discrepancy of Korobov's $p$-sets. Proc. Am. Math. Soc. 143, 5043-5057 (2015)

[3] Hinrichs, A.: Discrepancy, integration and tractability. In: Monte Carlo and Quasi-Monte Carlo Methods 2012, pp. 123-163, Springer Proc. Math. Stat., 65, Springer, Berlin (2016)

[4] Hinrichs, A., Oettershagen, J.: Optimal point sets for quasi-Monte Carlo integration of bivariate periodic functions with bounded mixed derivatives. In: Monte Carlo and Quasi-Monte Carlo Methods 2014, pp. 385-405, Springer Proc. Math. Stat., 163, Springer, Cham (2013)

[5] Hinrichs, A., Weyhausen, H.: Asymptotic behavior of average $L_{p}$-discrepancies. J. Complex. 28, 425-439 (2012)

[6] Hua, L.K., Wang, Y.: Applications of Number Theory to Numerical Analysis. Springer, Berlin (1981)

[7] Korobov, N.M.: Approximate calculation of repeated integrals by numbertheoretical methods (Russian). Dokl. Akad. Nauk SSSR (N.S.) 115, 1062-1065 (1957)

[8] Korobov, N.M.: On number-theoretic methods in approximate analysis (Russian). Probl. Numer. Math. Comp. Techn., Gosudarstv. Naučno-Tehn. Izdat. Mašinostr. Lit., Moscow, pp. 36-44 (1963)

[9] Lev, V.F.: On two versions of $L^{2}$-discrepancy and geometrical interpretation of diaphony. Acta Math. Hungar. 69(4), 281-300 (1995)

[10] Lidl, R., Niederreiter, H.: Finite Fields. Encyclopedia of Mathematics and its Applications, 20. Addison-Wesley Publishing Company, Advanced Book Program, Reading, MA, (1983); 2nd ed., Cambridge University Press (1997)

[11] Novak, E., Woźniakowski, H.: Intractability results for integration and discrepancy. J. Complex. 17, 388-441 (2001) 
[12] Novak, E., Woźniakowski, H.: Tractability of Multivariate Problems, Volume II: Standard Information for Functionals. European Mathematical Society, Zürich (2010)

[13] Sloan, I.H., Woźniakowski, H.: When are quasi-Monte Carlo algorithms efficient for high dimensional integrals? J. Complex. 14, 1-33 (1998)

[14] Weil, A.: On some exponential sums. Proc. Nat. Acad. Sci. USA 34, 204-207 (1948)

[15] Zinterhof, P.: Über einige Abschätzungen bei der Approximation von Funktionen mit Gleichverteilungsmethoden (German). Österr. Akad. Wiss. Math.Naturwiss. Kl. S.-B. II(185), 121-132 (1976)

\section{Josef Dick}

School of Mathematics and Statistics

The University of New South Wales

Sydney NSW 2052

Australia

e-mail: josef.dick@unsw.edu.au

\section{AiCKE HinRICHS}

Institut für Analysis

Johannes Kepler Universität Linz

Altenbergerstraße 69

4040 Linz

Austria

e-mail: aicke.hinrichs@jku.at

Friedrich Pillichshammer

Institut für Finanzmathematik und Angewandte Zahlentheorie

Johannes Kepler Universität Linz

Altenbergerstraße 69

4040 Linz

Austria

e-mail: friedrich.pillichshammer@jku.at

Received: 7 January 2020 